\title{
Nonlinear Dirac operator and quaternionic analysis.
}

\author{
Andriy Haydys* \\ Universität Bielefeld
}

May 30, 2008

\begin{abstract}
Properties of the Cauchy-Riemann-Fueter equation for maps between quaternionic manifolds are studied. Spaces of solutions in case of maps from a K3-surface to the cotangent bundle of a complex projective space are computed. A relationship between harmonic spinors of a generalized nonlinear Dirac operator and solutions of the CauchyRiemann-Fueter equation are established.
\end{abstract}

\section{Introduction.}

Nonlinear generalizations of the Dirac operator were known to physicists long ago [3] and appeared in the framework of the $\sigma$-model. Much later they were considered by mathematicians [22, 19] in the realm of the SeibergWitten theory. The basic idea of generalization is to replace the fibre of the spinor bundle $\mathbb{C}^{2} \cong \mathbb{H}$, i.e. the simplest hyperKähler manifold, by an arbitrary hyperKähler manifold with suitable symmetries.

On the other hand, Anselmi and Fre [1] generalized quaternionic analysis in the form of Fueter [13, 20] for maps between arbitrary hyperKähler manifolds. It is natural to expect that there is a connection between these

*this paper is based upon part of the author's thesis; partially supported by the grant "Gauge theory and exceptional geometry" (Universität Bielefeld) while preparing the final form 
two approaches, since in case of the flat source manifold harmonic spinors are exactly solutions to the Cauchy-Riemann-Fueter equation

$$
\frac{\partial u}{\partial x_{0}}-i \frac{\partial u}{\partial x_{1}}-j \frac{\partial u}{\partial x_{2}}-k \frac{\partial u}{\partial x_{3}}=0, \quad x \in \mathbb{H}, u: \mathbb{H} \rightarrow \mathbb{H} .
$$

One of the purposes of this paper is to establish a link between the generalized Dirac operator and quaternionic analysis.

The paper is organized as follows. In section 2 we study properties of the Cauchy-Riemann-Fueter equation for maps between quaternionic manifolds. In particular we show that its solutions are exactly those maps, whose differential has vanishing quaternion-linear component. Therefore we call such maps aholomorphic. Usually a hyperKähler manifold comes equipped with some symmetries. It turns out that certain symmetries of the target manifold force aholomorphic maps to be (anti)holomorphic (in the usual complex sense). This in turn allows to apply a well-developed technique of algebraic geometry to compute certain spaces of aholomorphic maps. In the last section we show that the nonlinear Dirac operator can be regarded as an analogue of the $\partial$-operator in complex geometry. Harmonic spinors are shown to be twisted (in an appropriate sense) aholomorphic maps.

\section{Aholomorphic maps.}

Algebraic preliminaries. Denote by $\mathbb{H}$ the $\mathbb{R}$-algebra of quaternions and by $S p(1)$ the group of quaternions of unitary length. Let $\left(U, J_{1}, J_{2}, J_{3}\right)$ and $\left(V, I_{1}, I_{2}, I_{3}\right)$ be quaternionic vector spaces. One can regard $U$ and $V$ as natural real $S p(1)$-representations. Further, denote by $W$ the standard $S p(1)$ representation given by the left multiplication on the space of quaternions $\mathbb{H}$. As usual, $\mathfrak{s p}(1)$ denotes the adjoint representation of $S p(1)$.

Proposition 2.1. Let $\operatorname{dim}_{\mathbb{H}} U=m, \operatorname{dim}_{\mathbb{H}} V=n$. We have the following decomposition into irreducible components:

$$
H_{\operatorname{com}}(U, V) \cong 4 m n \mathfrak{s p}(1) \oplus \mathbb{R}^{4 m n},
$$

where $\mathbb{R}^{4 m n}$ denotes the trivial $4 m n$-dimensional representation.

Proof. First observe, that a choice of quaternionic basis gives an isomorphism $U \cong W \otimes \mathbb{R}^{m}$ and similarly $V \cong W \otimes \mathbb{R}^{n}$. Since $W^{*} \cong W$ we get

$$
H o m_{\mathbb{R}}(U, V) \cong U^{*} \otimes V \cong W \otimes_{\mathbb{R}} W \otimes \mathbb{R}^{m n}
$$


Further, since $\bar{W} \cong W$ we have $\left(W \otimes_{\mathbb{R}} W\right)_{\mathbb{C}} \cong W_{\mathbb{C}} \otimes_{\mathbb{C}} W_{\mathbb{C}} \cong W \otimes W \otimes \mathbb{C}^{2} \cong$ $\left(S^{2} W \oplus \mathbb{C}\right) \otimes \mathbb{C}^{2} \cong\left(\mathfrak{s p}(1)_{\mathbb{C}} \oplus \mathbb{C}\right) \otimes \mathbb{C}^{2}$ and the statement follows.

Our next aim is to find subspaces $B_{ \pm} \subset \operatorname{Hom}_{\mathbb{R}}(U, V)$ that give the decomposition (11).

Consider a linear map $C: \operatorname{Hom}_{\mathbb{R}}(U, V) \rightarrow \operatorname{Hom}_{\mathbb{R}}(U, V)$,

$$
C(A)=I_{1} A J_{1}+I_{2} A J_{2}+I_{3} A J_{3} .
$$

A direct computation shows that $C$ satisfies the equation $C^{2}+2 C-3=0$. Consequently, $C$ has two eigenvalues 1 and -3 and we have the decomposition

$$
\operatorname{Hom}_{\mathbb{R}}(U, V)=B_{+} \oplus B_{-},
$$

where $B_{+}$(resp. $B_{-}$) denotes the eigenspace corresponding to the eigenvalue 1 (resp. -3 ). Observe that since $C$ is $S p(1)$-invariant, the subspaces $B_{ \pm}$are also $S p(1)$-invariant.

The trivial $S p(1)$-subrepresentation of $\operatorname{Hom}_{\mathbb{R}}(U, V)$ is by definition the space $H_{\text {om }}(U, V)$ of quaternion-linear maps. It is straightforward to check the inclusions $\operatorname{Hom}_{\mathbb{H}}(U, V) \subset B_{-}, \operatorname{Hom}_{\mathbb{H}}(U, V) \otimes \mathbb{R}^{3} \subset B_{+}$, where the second one is given by

$$
A_{1} \otimes e_{1}+A_{2} \otimes e_{2}+A_{3} \otimes e_{3} \mapsto I_{1} A_{1}+I_{2} A_{2}+I_{3} A_{3} .
$$

By dimension counting we conclude that these inclusions are in fact isomorphisms. Since $B_{+}$is $S p(1)$-invariant and complementary to the trivial $4 m n$-dimensional representation, it must be isomorphic to $4 m n \mathfrak{s p}(1)$. We summarize the above considerations in the following proposition.

Proposition 2.2. The eigenspace $B_{-}$of the linear map $C$ (2) corresponding to the eigenvalue -3 consists of quaternion-linear maps and we have the following decomposition

$$
H o m_{\mathbb{R}}(U, V) \cong H o m_{\mathbb{H}}(U, V) \oplus B_{+} .
$$

Definition 2.3. We say that a linear map $A \in H_{0} m_{\mathbb{R}}(U, V)$ between two quaternionic vector spaces $\left(U, J_{1}, J_{2}, J_{3}\right)$ and $\left(V, I_{1}, I_{2}, I_{3}\right)$ is aquaternionic if

$$
I_{1} A J_{1}+I_{2} A J_{2}+I_{3} A J_{3}=A \text {. }
$$

Corollary 2.4. An $\mathbb{R}$-linear map is aquaternionic if and only if its quaternion-linear component vanishes. 
Aholomorphic maps. Let $\left(X, J_{1}, J_{2}, J_{3}\right)$ and $\left(M, I_{1}, I_{2}, I_{3}\right)$ be almost hypercomplex manifolds and $u: X \rightarrow M$ be a smooth map. Then the differential $u_{*}$ is pointwise an $\mathbb{R}$-linear map between quaternionic vector spaces T.X and T.M.

Definition 2.5. We say that a map $u: X \rightarrow M$ is aholomorphic, if it satisfies the Cauchy-Riemann-Fueter equation

$$
I_{1} u_{*} J_{1}+I_{2} u_{*} J_{2}+I_{3} u_{*} J_{3}=u_{*} \text {. }
$$

Theorem 2.6. A map $u: X \rightarrow M$ is aholomorphic if and only if the quaternion-linear component of its differential vanishes at each point.

Aholomorphic maps were studied under a variety of different names. They naturally arise in the supersymmetric gauged $\sigma$-model and appeared in physical literature [1, 11] for the first time as "triholomorphic maps" or "hyperinstantons". Such maps naturally appear in higher-dimensional gauge theory [9] and were also studied by Chen [7], Chen and Li [8] ("quaternionic maps"), Wang [23] ("triholomorphic maps"). Joyce [16] ("q-holomorphic functions") considered the case of the flat target manifold $\mathbb{H}$. Equation (3) was known long ago and was introduced in 1934 by Fueter [13] ("regular functions") for the simplest case of maps $u: \mathbb{H} \rightarrow \mathbb{H}$ in his attempts to construct a quaternionic analogue of the theory of complex holomorphic maps. An extensive exposition of the theory can be found in [20].

In the author's opinion the proposed term aholomorphic map better reflects the properties of maps satisfying equation (3), namely the fact that the differential of solutions to (3) has a vanishing quaternion-linear component.

A reader can find examples of aholomorphic maps in the above mentioned sources. Other examples will appear below.

Observe that the Cauchy-Riemann-Fuether equation (3) is elliptic only in case when a source manifold $X$ is four-dimensional. We will concentrate on this case below.

We now consider aholomorphic maps between hyperKähler manifolds. A Riemannian $4 n$-dimensional manifold $M$ is called hyperKähler if the holonomy group is a subgroup of $S p(n)$. In other words, a Riemannian manifold $(M, g)$ is hyperKähler if it admits three covariantly constant complex structures $I_{1}, I_{2}, I_{3}$ with quaternionic relations

$$
I_{1} I_{2}=-I_{2} I_{1}=I_{3}, \quad I_{1}^{2}=I_{2}^{2}=I_{3}^{2}=-i d,
$$


compatible with the Riemannian structure: $g\left(I_{l} \cdot, I_{l} \cdot\right)=g(\cdot, \cdot), l=1,2,3$. Let $\omega_{l}$ denote the Kähler 2 -form corresponding to $I_{l}$.

Proposition 2.7. Suppose $X$ and $M$ are both hyperKähler manifolds. If $X$ is also compact and 4-dimensional (i.e. $X$ is a torus or a K3 surface), then for any smooth map $u: X \rightarrow M$ the following identity holds

$$
\frac{1}{2}\left\|u_{*}\right\|_{L_{2}}^{2}=\frac{1}{4}\left\|u_{*}-C\left(u_{*}\right)\right\|_{L_{2}}^{2}-\sum_{l=1}^{3} \int_{X} \omega_{l}^{X} \wedge u^{*} \omega_{l}^{M} .
$$

This proposition was essentially proven by Chen and Li [ㅁ, Proposition 2.2]. Formula (4) immediately follows from the result of Chen and $\mathrm{Li}$, once you observe that each Kähler form on $X$ is self-dual and that the induced scalar product on $\Lambda^{2} \mathbb{R}^{4}$ is given by the sequence

$$
\Lambda^{2} \mathbb{R}^{4} \otimes \Lambda^{2} \mathbb{R}^{4} \stackrel{* \otimes i d}{\longrightarrow} \Lambda^{2} \mathbb{R}^{4} \otimes \Lambda^{2} \mathbb{R}^{4} \stackrel{\cdot \wedge \cdot}{\longrightarrow} \Lambda^{4} \mathbb{R}^{4} \cong \mathbb{R} .
$$

Corollary 2.8 (Vanishing theorem). Let $X$ and $M$ be as in Proposition 2.7. If the cohomology class of each Kähler form $\omega_{l}^{M}$ on $M$ vanishes, then any aholomorphic map $u: X \rightarrow M$ is constant.

Let $\mathcal{I}=\mathcal{I}_{M}$ denote the trivial 3-dimensional subspace of $\Gamma(\operatorname{End}(T M))$ spanned by $I_{1}, I_{2}$ and $I_{3}$ naturally identified with $\operatorname{Im} \mathbb{H}=\mathfrak{s p}(1)$.

Definition 2.9. An isometric action of the group $S p(1)$ (or $S O(3)$ ) on a hyperKähler manifold $M$ is called permuting if the subspace $\mathcal{I}$ is preserved and the induced action on $\mathcal{I}$ is the adjoint one.

The hypothesis of Corollary 2.8 is automatically satisfied for hyperKähler manifolds that admit a permuting action of $S p(1)$ or $S O(3)$ [6]. Such actions will play a crucial role in Section 3 . The class of hyperKähler manifolds that admit a permuting action is quite wide and includes a lot of interesting examples: $\mathbb{H}^{n}$ with the flat metric and its hyperKähler reductions with respect to the zero value of momentum map; different moduli spaces, obtained as infinite-dimensional hyperKähler reductions and in particular moduli spaces of framed instantons [18] over $\mathbb{R}^{4}$ and monopoles [2]. For any quaternionic Kähler manifold with positive scalar curvature Swann [21] constructed a hyperKähler manifold with permuting action of $\mathbb{H}^{*} \supset S p(1)$. 
Let us consider a lager class of target hyperKähler manifolds. Namely suppose that $\left(M, I_{1}, I_{2}, I_{3}\right)$ admits only an action of $S^{1}$ that fixes one complex structure, say $I_{1}$, and rotates the other two, i.e.

$$
\left(L_{z}\right)_{*} I_{1}=I_{1}\left(L_{z}\right)_{*}, \quad\left(L_{z}\right)_{*} I_{w}\left(L_{\bar{z}}\right)_{*}=I_{z w}
$$

where $L_{z}: M \rightarrow M$ denotes the left shift by $z \in S^{1}, w=a+b i$ is a complex number of unitary length, $I_{w}=a I_{2}+b I_{3}$.

Proposition 2.10. Let $X$ be a compact hyperKähler 4-dimensional manifold. Assume that the target hyperKähler manifold $M$ admits an isometric action of $S^{1}$ that fixes $I_{1}$ while rotating $I_{2}$ and $I_{3}$. Then a map $u: X \rightarrow M$ is aholomorphic if and only if it is $\left(J_{1}, I_{1}\right)$-antiholomorphic.

Proof. First notice that existence of an isometric $S^{1}$-action that fixes $I_{1}$ and rotates the other two complex structures implies that $\omega_{2}^{M}$ and $\omega_{3}^{M}$ are exact [15. Indeed, denote by $K$ the Killing vector field of the $S^{1}$-action and by $\mathcal{L}_{K}$ the Lie derivative. It follows from (5) that $\mathcal{L}_{K} \omega_{2}^{M}=\omega_{3}^{M}$. Applying the Cartan formula, one gets $\omega_{3}^{M}=\mathcal{L}_{K} \omega_{2}^{M}=d\left(\imath_{K} \omega_{2}^{M}\right)$. Therefore equality (4) takes the following form:

$$
\frac{1}{2}\left\|u_{*}\right\|_{L_{2}}^{2}=\frac{1}{4}\left\|u_{*}-C\left(u_{*}\right)\right\|_{L_{2}}^{2}-\int_{X} \omega_{1}^{X} \wedge u^{*} \omega_{1}^{M} .
$$

Further, since the action of $S^{1}$ is isometric, the energy functional

$$
E(u)=\frac{1}{2}\left\|u_{*}\right\|_{L_{2}}^{2}=\frac{1}{2} \int_{X}\left\|u_{*}\right\|^{2} \operatorname{dvol}_{X}, \quad u: X \rightarrow M
$$

is $S^{1}$-invariant.

Suppose now that $u$ is aholomorphic. In particular, $u$ is an absolute minimum of the energy functional (17) within its homotopy class $\alpha=[u]$. Then for each $z \in S^{1}$ the map $u^{z}=L_{z} \circ u$ lies in the same homotopy class $\alpha$ and is also an absolute minimum of the energy functional within $\alpha$. From equation (6) we conclude that $u^{z}$ must be aholomorphic:

$$
I_{1} u_{*}^{z} J_{1}+I_{2} u_{*}^{z} J_{2}+I_{3} u_{*}^{z} J_{3}=u_{*}^{z} \text {. }
$$

The above equation can be rewritten as

$$
I_{1} u_{*} J_{1}+\left(L_{\bar{z}}\right)_{*} I_{2}\left(L_{z}\right)_{*} u_{*} J_{2}+\left(L_{\bar{z}}\right)_{*} I_{3}\left(L_{z}\right)_{*} u_{*} J_{3}=u_{*} .
$$


In particular, for $z=-1$ we get $I_{1} u_{*} J_{1}-I_{2} u_{*} J_{2}-I_{3} u_{*} J_{3}=u_{*}$. Since $u$ satisfies Cauchy-Riemann-Fueter equation (3), we obtain $I_{1} u_{*} J_{1}=u_{*}$, i.e. $u$ is $\left(J_{1}, I_{1}\right)$-antiholomorphic. On the other hand, it easily follows from the definition that any antiholomorphic map is also aholomorphic.

An example of the circle action preserving one complex structure and rotating the other two is the standard fiberwise action on the cotangent bundle $\mathrm{T}^{*} \mathbb{P}^{n}$ of the complex projective space $\mathbb{P}^{n}$ equipped with the Calabi metric. More generally, Kaledin [17] and independently Feix [10] constructed such metrics on a neighborhood of the zero section in $T^{*} Z$ for real-analytic Kähler manifolds $Z$.

Example 2.11 (Aholomorphic maps from a K3-surface into $\mathrm{T}^{*} \mathbb{P}^{n}$ ). 11t follows from Proposition 2.10 that any aholomorphic map $u: X \rightarrow \mathrm{T}^{*} \mathbb{P}^{n}$ must be $\left(J_{1}, I_{1}\right)$-antiholomorphic. The standard antiholomorphic automorphism of $\mathbb{P}^{n}$ induces an $I_{1}$-antiholomorphic automorphism on $\mathrm{T}^{*} \mathbb{P}^{n}$ and therefore we have a natural bijection between the spaces of holomorphic and antiholomorphic maps into $\mathrm{T}^{*} \mathbb{P}^{n}$. Further, each holomorphic map $u: X \rightarrow \mathrm{T}^{*} \mathbb{P}^{n}$ naturally decomposes into the projection $\varphi: X \rightarrow \mathbb{P}^{n}$ and a holomorphic section $s \in \Gamma\left(\varphi^{*} \mathrm{~T}^{*} \mathbb{P}^{n}\right)$. Each nonconstant holomorphic map $\varphi$ into the projective space can be obtained as a morphism associated to a linear system of a positive base point free divisor $D$ (see [14 for example). Then $\varphi^{*} \mathcal{O}_{\mathbb{P}^{n}}(1)=\mathcal{O}_{X}(D)$ and the pull-back to $X$ of the short exact sequence dual to the Euler one has the following form:

$$
0 \rightarrow \varphi^{*} \mathrm{~T}^{*} \mathbb{P}^{n} \rightarrow(n+1) \mathcal{O}_{X}(-D) \rightarrow \mathcal{O}_{X} \rightarrow 0
$$

It follows that the lift $s$ must be the zero section. Therefore our problem reduces to description of the space of holomorphic maps $X \rightarrow \mathbb{P}^{n}$.

From now on we assume that $X$ is a K3-surface. Let a map $\varphi=\varphi_{D}$ : $X \rightarrow \mathbb{P}^{n}$ be given by the complete linear system $|D|=\mathbb{P}\left(H^{0}\left(\mathcal{O}_{X}(D)\right)\right)$ with empty base locus of a positive divisor $D$. Since $D^{2}$ is the degree of $\varphi$ we may assume $D^{2} \geq 0$. It turns out that base point free divisors $D$ on a K3-surface admit a purely numeric characterization at least if $D$ is big $\left(D^{2}>0\right)$. Indeed, for a big divisor $D$ the associated morphism $\varphi: X \rightarrow \mathbb{P}^{n}$ is generically finite. It follows that $\operatorname{dim} \varphi(X)=2$ and by Bertini's theorem [4] the divisor $D$ is linearly equivalent to a smooth irreducible curve $C$ with

\footnotetext{
${ }^{1}$ I am grateful to I.Panin for helpful discussions on this example
} 
$p_{a}(C)=1+C^{2} / 2=1+D^{2} / 2>1$. It follows that $D$ is nef (numerically effective) and by assumption $D$ is also big $\left(D^{2}>0\right)$. On the other hand, the complete linear system $|D|$ of a nef and big divisor $D$ has a non-empty base locus if and only if $D=k E+R$, where $R$ is a smooth rational curve $\left(R^{2}=-2\right), E$ is a smooth elliptic curve $\left(E^{2}=0\right), E \cdot R=1$ and $k \geq 2[12$. In case when $D$ is big, nef and base point free the associated morphism is a map into the complex projective space of dimension $N=1+D^{2} / 2$.

Now fix a positive integer $n$. Let $\varphi: X \rightarrow \mathbb{P}^{n}$ be a holomorphic map and $k$ be the dimension of the projective span of $\varphi(X)$, that is $\varphi$ decomposes as $i \circ \psi$, where $i: \mathbb{P}^{k} \hookrightarrow \mathbb{P}^{n}$ is a standard embedding. Then $\psi$ is a morphism given by a projective basis of a linear $k$-dimensional system of $D$ with $k \leq N=\operatorname{dim}|D|=1+D^{2} / 2$. One can describe $\psi$ equivalently as the composition of a morphism $\varphi_{D}: X \rightarrow \mathbb{P}^{N}$, given by a projective basis of the complete linear system $|D|$, and a projection $\pi: \mathbb{P}^{N} \backslash \mathbb{P}^{N-k-1} \rightarrow \mathbb{P}^{k}$ with respect to a subspace $\mathbb{P}^{N-k-1} \hookrightarrow \mathbb{P}^{N}$ that does not intersect the image of $\varphi_{D}$. Denote by $\mathbb{P} V_{N-k}\left(\mathbb{C}^{N+1}\right)$ the projective Stiefel manifold, $\mathbb{P V}_{N-k}\left(\mathbb{C}^{N+1}\right) \stackrel{p r}{\longrightarrow}$ $G r_{N-k}\left(\mathbb{C}^{N+1}\right)$. Then the space $\Psi(D, k)$ of all holomorphic maps $\psi: X \rightarrow \mathbb{P}^{k}$ that can be obtained by a choice of $k+1$ linearly independent sections of $\mathcal{O}_{X}(D)$ without base locus is the Zariski-open set

$$
p r^{-1}\left\{V \in G r_{N-k}\left(\mathbb{C}^{N+1}\right) \mid[V] \cap \varphi_{D}(X)=\emptyset\right\} \subset \mathbb{P V} V_{N-k}\left(\mathbb{C}^{N+1}\right) .
$$

In particular, $\Psi(D, k)$ is connected.

Observe also that $\mathbb{P}^{N} \backslash \mathbb{P}^{N-k-1}$ is the total space of a vector bundle over $\mathbb{P}^{k} \hookrightarrow \mathbb{P}^{N}$. Therefore $\pi \circ \varphi_{D}$ is homotopic to $\varphi_{D}$. It follows that the spaces $\Psi(D, k)$ and $\Psi\left(D^{\prime}, k^{\prime}\right)$ lie in the same component of $\operatorname{Map}\left(X, \mathbb{P}^{n}\right)$ if and only if $D=D^{\prime}$.

Summing up the above considerations we get the following result.

Proposition 2.12. Let $X$ be a K3-surface and $n$ be a positive integer. Then the space of all nonconstant aholomorphic maps $u: X \rightarrow \mathrm{T}^{*} \mathbb{P}^{n}$ (equivalently, the space of all holomorphic maps $\varphi: X \rightarrow \mathbb{P}^{n}$ ) is the stratified space

$$
\bigsqcup_{D, k} \Psi(D, k) \times G r_{k+1}\left(\mathbb{C}^{n+1}\right),
$$

where $D$ denotes a divisor class on $X$ such that the base locus of the complete linear system $|D|$ is empty; $k$ is an integer, $1 \leq k \leq n ; \Psi(D, k)$ is a Zariskiopen subset (8) of the projective Stiefel manifold $\mathbb{P V}_{N-k}\left(\mathbb{C}^{N+1}\right), N=1+$ $D^{2} / 2$. 
The subsets $\Psi(D, k) \times G r_{k+1}\left(\mathbb{C}^{n+1}\right)$ and $\Psi\left(D^{\prime}, k^{\prime}\right) \times G r_{k+1}\left(\mathbb{C}^{n+1}\right)$ lie in the same component of $\operatorname{Map}\left(X, \mathrm{~T}^{*} \mathbb{P}^{n}\right)$ if and only if $D=D^{\prime}$.

Observe that any holomorphic map $X \rightarrow \mathbb{P}^{n}$ can be obtained from a morphism $\varphi_{D}$, given by a primitive divisor $D$, composing it with holomorphic maps between complex projective spaces. In the examples below we will indicate only the primitive divisor classes.

For a $\mathrm{K} 3$-surface $X$ the Euclidean lattice $\left(H^{2}(X ; \mathbb{Z}), \cup\right)$ is isomorphic to $-2 E_{8} \oplus 3 H$ and is of rank 22. Notice that by the Torelli theorem a K3surface can be specified by its period point (see [4 for details). The natural map $c_{1}: \operatorname{Pic}(X) \rightarrow H^{2}(X, \mathbb{Z})$ is injective and the rank $\rho$ of its image, the Neron-Severi group $N S(X)$, can take any integer value between 0 and 20 . We shall consider some examples for small values of $\rho$ (the examples are taken from [5]).

1) $\rho=1, N S(X) \cong\left\langle 2 k^{2}\right\rangle$. We have just one primitive divisor class $D$ with $D^{2}=2 k^{2}$. The complete linear system is base point free and we get a regular holomorphic map $\varphi_{D}: X \rightarrow \mathbb{P}^{1+k^{2}}$.

2) $\rho=2, N S(X) \cong H$. In this case the two generators $E_{1}$ and $E_{2}$ are elliptic curves. The complete linear system $\left|E_{i}\right|$ is base point free [12] and defines a regular map $\varphi_{E_{i}}: X \rightarrow \mathbb{P}^{1}$. Any other positive primitive divisor class has the form $D=p E_{1}+q E_{2}$, where $p$ and $q$ are positive coprime integers. The base locus of $D$ is empty and therefore we get a map $\varphi_{D}: X \rightarrow \mathbb{P}^{2 p q}$.

3) $\rho=2, N S(X) \cong\left(\begin{array}{rr}2 & 1 \\ 1 & -2\end{array}\right)$. Let $C$ and $R$ be generators, $C^{2}=2, R^{2}=$ $-2, C \cdot R=2$. It is easy to check that there are no divisors with vanishing selfintersection number. A divisor $D=p C+q R$ is nef iff $p \geq q$. In this case $D$ is also big. Consequently, for coprime $p$ and $q, p \geq q$ the divisor $D$ is primitive with empty base locus and we get a holomorphic map $\varphi_{D}: X \rightarrow \mathbb{P}^{1+p^{2}+2 p q-q^{2}}$.

\section{$3 \quad$ Harmonic spinors as twisted aholomorphic maps.}

In this section we establish connection between aholomorphic maps and (generalized) harmonic spinors. Before proceeding let us briefly outline the definition of the (generalized) Dirac operator in a form suitable for our purposes. Details can be found in the paper of Pidstrygach [19], where the Dirac operator is defined in a slightly more general context. 
Algebraic preliminaries. Recall that the group $\operatorname{Spin}(4)$ is isomorphic to the product of two copies of $S p(1)$ :

$$
\operatorname{Spin}(4)=S p_{+}(1) \times S p_{-}(1)
$$

where we use subscripts " \pm " to distinguish between different copies. The isomorphism $\operatorname{Spin}(4) / \pm 1 \cong S O(4)$ is given by

$$
\left(q_{+}, q_{-}\right) \mapsto B_{q_{+}, q_{-}}: \mathbb{H} \rightarrow \mathbb{H}, \quad B_{q_{+}, q_{-}} h=q_{-} h \bar{q}_{+} .
$$

Recall that $W$ denotes the $S p(1)$-representation on $\mathbb{H}$ by multiplication on the left. Further, $\mathbb{R}^{4}$ denotes the standard $S O(4)$-representation. The following homomorphism of $\operatorname{Spin}(4)=S p(1)_{+} \times S p_{-}(1)$-representations

$$
\mathbb{R}^{4} \otimes W^{+} \rightarrow W^{-}, \quad h_{1} \otimes h_{2} \mapsto h_{1} h_{2},
$$

is called the Clifford multiplication, where $W^{ \pm}$denote the representations induced by $S p_{ \pm}(1)$.

Let $\left(V, I_{1}, I_{2}, I_{3}\right)$ be a quaternionic vector space. We identify the subspace $\operatorname{span}\left(I_{1}, I_{2}, I_{3}\right) \subset \operatorname{End}_{\mathbb{R}}(V)$ with $\operatorname{Im} \mathbb{H}=\mathfrak{s p}(1)$.

Lemma 3.1. Let $V$ be a real representation of the group $S p(1)$ such that the induced action on $\operatorname{End}_{\mathbb{R}}(V)$ preserves $\operatorname{span}\left(I_{1}, I_{2}, I_{3}\right)$. Assume that the induced representation on $\operatorname{span}\left(I_{1}, I_{2}, I_{3}\right)$ coincides with the adjoint one. Then

$$
V_{\mathbb{C}} \cong W \otimes E
$$

where $E$ is an $S p(1)$-representation.

The proof is a straightforward calculation of the actions of $i, j, k \in S p(1)$ on $\mathbb{C} \otimes_{\mathbb{R}} V \cong \mathbb{C}^{2} \otimes_{\mathbb{C}} V_{I_{1}}$, where $V_{I_{1}}$ denotes the complex vector space $\left(V, I_{1}\right)$.

From (10) one obtains a variant of the Clifford multiplication:

$$
\mathbb{R}_{\mathbb{C}}^{4} \otimes V_{\mathbb{C}} \cong \mathbb{R}_{\mathbb{C}}^{4} \otimes W^{+} \otimes E \rightarrow W^{-} \otimes E
$$

Observe that the homomorphism (11) induces an homomorphism between real parts:

$$
\mathbb{R}^{4} \otimes V \rightarrow\left[W^{-} \otimes E\right]_{r}
$$

The nonlinear Dirac operator. From now on $X^{4}$ is a smooth closed oriented Riemannian manifold. We also assume that $X$ is a spin manifold 
with a $\operatorname{Spin}(4)$-principal bundle $\pi: P \rightarrow X$. The assumption that $X$ is spin is not essential, since the bundle $P$ can be replaced by a $\operatorname{Spin}^{c}(4)$-bundle, however the exposition becomes a bit clearer in case of spin manifolds.

Let $\left(M, I_{1}, I_{2}, I_{3}\right)$ be a hyperKähler manifold with permuting action of $S p(1)$ (see Definition 2.9). Then the group $\operatorname{Spin}(4)$ acts on $M$ via the homomorphism $\operatorname{Spin}(4) \rightarrow S p_{+}(1)$ and we get the associated fibre bundle:

$$
\mathbb{M}=P \times_{\operatorname{Spin}(4)} M .
$$

Observe that in case $M=\mathbb{H}$ with the standard action of $S p(1)$ one gets the usual positive spinor bundle $\mathcal{W}^{+}$. Therefore sections of $\mathbb{M}$ are called (generalized) spinors. Notice that the space of spinors can be naturally identified with the space of equivariant maps:

$$
\Gamma(\mathbb{M}) \cong \operatorname{Map}^{\operatorname{Spin}(4)}(P, M) .
$$

The Levi-Civita connection on $P$ determines the covariant derivative:

$$
\nabla_{\mathrm{v}} u=u_{*}(\hat{\mathrm{v}}), \quad u \in \operatorname{Map}^{\operatorname{Spin}(4)}(P, M)
$$

where $\hat{\mathrm{v}}$ denotes the horizontal lift of $\mathrm{v} \in T X$. In other words we get a map

$$
\nabla: \operatorname{Map}^{\operatorname{Spin}(4)}(P, M) \rightarrow \Gamma\left(T^{*} X \otimes \pi_{!}\left(u^{*} T M\right)\right),
$$

where $\pi_{!}\left(u^{*} T M\right) \rightarrow X$ denotes the factor of $u^{*} T M \rightarrow P$ by the group action.

Remark 3.2. Strictly speaking, the operator $\nabla$ is not well-defined, since its range depends on the element of the domain. However one can define $\nabla$ as a section of a certain vector bundle as follows. Consider the evaluation map $e v: \operatorname{Map}^{\operatorname{Spin}(4)}(P, M) \times P \rightarrow M,(u, p) \mapsto u(p)$. Then one gets the following diagram

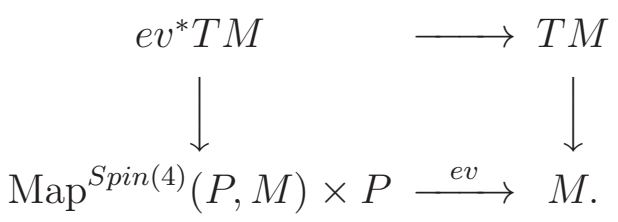

Dividing the first column by the group action one gets a vector bundle $\mathcal{E}$ over an the infinite dimensional space $\operatorname{Map}^{\operatorname{Spin}(4)}(P, M) \times X$; the restriction of $\mathcal{E}$ to $\{u\} \times X$ coincides with $\pi_{!}\left(u^{*} T M\right)$. Then $\nabla$ is well-defined as a section of $\mathcal{E} \rightarrow \operatorname{Map}^{\operatorname{Spin}(4)}(P, M) \times X$.

In order to keep the exposition clear, we will not keep to the above formalism of vector bundles over infinite-dimensional spaces. 
The tangent space of $M$ has a natural structure of quaternionic vector space at each point. Since we have a permuting action of $S p(1)$ on $M$, we get from the Proposition 3.1 that $T_{\mathbb{C}} M \cong W^{+} \otimes \tilde{E}$, where $\tilde{E}$ is a complex vector bundle over $M$ with an action of $S p(1)$ ( $\tilde{E}$ coicides with $\left(T M, I_{1}\right)$ as a complex vector bundle, however the action of $S p(1)$ is different from the induced one). Consequently, we get the Clifford multiplication

$$
C l: T^{*} X \otimes \pi_{!}\left(u^{*} T M\right) \rightarrow\left[\mathcal{W}^{-} \otimes E\right]_{r},
$$

where $E=\pi_{!}\left(u^{*} \tilde{E}\right)$ and $\mathcal{W}^{-}$is the negative spinor bundle over $X$.

Definition 3.3 ([19]). The first order differential operator $\mathcal{D}$ defined by the sequence

$$
\mathcal{D}: \Gamma(\mathbb{M}) \stackrel{\nabla}{\longrightarrow} \Gamma\left(T^{*} X \otimes \pi_{!}\left(u^{*} T M\right)\right) \stackrel{C l}{\longrightarrow} \Gamma\left(\left[\mathcal{W}^{-} \otimes E\right]_{r}\right)
$$

is called a (generalized) Dirac operator.

Definition 3.4. A spinor $u$ such that $\mathcal{D} u=0$ is called harmonic.

Remark 3.5. The Dirac operator is well-defined as a section of a vector bundle over an infinite-dimensional space similarly as the covariant derivative (see Remark 3.2) and it is a Fredholm section [19]. In case when the fibre $M$ of the spinor bundle is a copy of quaternions with the standard action of $S p(1)$ one recovers the usual linear Dirac operator.

Remark 3.6. Notice that if the target hyperKähler manifold admits a permuting action of $S O(3)$ rather then $S p(1)$ one needs just the principal $S O(4)-$ bundle of orthonormal frames rather then its Spin(4)-lifting to define the Dirac operator. A well-known example in classical theory is $d^{+}+d^{*}$ : $\Omega^{1}(X) \rightarrow \Omega_{+}^{2}(X) \oplus \Omega^{0}(X)$.

One can also define a Dirac operator with the help of a $\operatorname{Spin}^{c}(4)$-structure. In this case the target manifold $M$ is required to carry a triholomorphic action of $S^{1}$ commuting with the permuting action of $S p(1)$.

The Levi-Civita connection splits $T P$ into horizontal and vertical bundles: $T P \cong \mathcal{H} \oplus \mathcal{V}$. Since we have a natural projection $p r: P \rightarrow P_{S O}$ onto the principal bundle of orthonormal frames of $X$, the horizontal bundle $\mathcal{H}$ has a natural quaternionic structure $\left(J_{1}, J_{2}, J_{3}\right)$, which is defined as follows: a point $p \in P$ determines an orthonormal basis $\mathrm{v}=\operatorname{pr}(p)=\left(\mathrm{v}_{0}, \mathrm{v}_{1}, \mathrm{v}_{2}, \mathrm{v}_{3}\right)$ of 
$T_{\pi(p)} X$ and consequently a basis $\hat{\mathrm{v}}$ of $\mathcal{H}_{p}$; then $\left(J_{1}, J_{2}, J_{3}\right)$ is defined as the unique quaternionic structure 2 such that $\hat{\mathrm{v}}_{l}=-J_{l} \hat{\mathrm{v}}_{0}$ for $l=1,2,3$.

Theorem 3.7. For a map $u \in \operatorname{Map}^{\operatorname{Spin}(4)}(P, M)$ denote by $u_{*}^{h}$ the restriction of the differential $u_{*}$ to the horizontal subbundle $\mathcal{H} \subset T P$. Then the spinor $u$ is harmonic if and only if the Cauchy-Riemann-Fueter-type equation holds:

$$
I_{1} u_{*}^{h} J_{1}+I_{2} u_{*}^{h} J_{2}+I_{3} u_{*}^{h} J_{3}=u_{*}^{h} .
$$

Proof. Pick a point $p \in P$ and denote by $\mathrm{v}=\operatorname{pr}(p)$ the basis of $T X$ at the point $x=\pi(p)$ as before. Let $u \in \operatorname{Map}^{\operatorname{Sin}(4)}(P, M)$ be a harmonic spinor, $m=u(p) \in M$.

The pull-back of the Clifford multiplication (12) to $P$ can be described by the following sequence (see (10), (11) ):

$$
\begin{aligned}
\mathbb{R}^{4} \otimes u^{*} T M & \hookrightarrow\left(\mathbb{R}^{4} \otimes u^{*} T M\right)_{\mathbb{C}} \cong \mathbb{R}_{\mathbb{C}}^{4} \otimes u^{*} T_{\mathbb{C}} M \\
& \cong\left(W^{-} \otimes W^{+}\right) \otimes\left(W^{+} \otimes T M\right) \rightarrow W^{-} \otimes T M .
\end{aligned}
$$

Notice that the image of the above map lies automatically in the real part of $W^{-} \otimes T M$. Further, $\mathbb{R}^{4}$ and $W^{ \pm}$are isomorphic to $\mathbb{H}$ as vector spaces. Then the homomorphism (13) is the following map:

$$
h \otimes w \mapsto h \cdot 1 \otimes w-h \cdot j \otimes I_{2} w, \quad h \in \mathbb{H}, w \in T M .
$$

Observe that $h=1$ (resp. $i, j, k$ ) corresponds to the horizontal lift of $\mathrm{v}_{0}$ (resp. $\left.\mathrm{v}_{1}, \mathrm{v}_{2}, \mathrm{v}_{3}\right)$. According to the definition of the Dirac operator take $h=1, w=\nabla_{\hat{\mathrm{v}}_{0}} u=u_{*}\left(\hat{\mathrm{v}}_{0}\right)=u_{*}^{h}\left(\hat{\mathrm{v}}_{0}\right) ; h=i, w=u_{*}^{h}\left(\hat{\mathrm{v}}_{1}\right) \ldots$ and sum up the result. It follows that $u$ is harmonic at the point $x$ if and only if the following equation holds

$$
\begin{gathered}
\left(1 \otimes u_{*}^{h}\left(\hat{\mathrm{v}}_{0}\right)-j \otimes I_{2} u_{*}^{h}\left(\hat{\mathrm{v}}_{0}\right)\right)+\left(i \otimes u_{*}^{h}\left(\hat{\mathrm{v}}_{1}\right)-k \otimes I_{2} u_{*}^{h}\left(\hat{\mathrm{v}}_{1}\right)\right)+ \\
+\left(j \otimes u_{*}^{h}\left(\hat{\mathrm{v}}_{2}\right)+1 \otimes I_{2} u_{*}^{h}\left(\hat{\mathrm{v}}_{2}\right)\right)+\left(k \otimes u_{*}^{h}\left(\hat{\mathrm{v}}_{3}\right)+i \otimes I_{2} u_{*}^{h}\left(\hat{\mathrm{v}}_{3}\right)\right)=0 .
\end{gathered}
$$

After a simplification one gets

$$
\begin{aligned}
& 1 \otimes\left(u_{*}^{h}\left(\hat{\mathrm{v}}_{0}\right)+I_{1} u_{*}^{h}\left(\hat{\mathrm{v}}_{1}\right)+I_{2} u_{*}^{h}\left(\hat{\mathrm{v}}_{2}\right)+I_{3} u_{*}^{h}\left(\hat{\mathrm{v}}_{3}\right)\right) \\
&+j \otimes\left(-I_{2} u_{*}^{h}\left(\hat{\mathrm{v}}_{0}\right)+I_{3} u_{*}^{h}\left(\hat{\mathrm{v}}_{1}\right)+u_{*}^{h}\left(\hat{\mathrm{v}}_{2}\right)-I_{1} u_{*}^{h}\left(\hat{\mathrm{v}}_{3}\right)\right)=0 .
\end{aligned}
$$

\footnotetext{
${ }^{2}$ the basis v can be viewed as an isomorphism $T_{x} X \cong \mathbb{H}$; then the quaternionic structure on $\mathbb{H}$, compatible with the isomorphism (9), is the right one
} 
It is easy to see that (14) is equivalent to the single equation $u_{*}^{h}\left(\hat{\mathrm{v}}_{0}\right)+$ $I_{1} u_{*}^{h}\left(\hat{\mathrm{v}}_{1}\right)+I_{2} u_{*}^{h}\left(\hat{\mathrm{v}}_{2}\right)+I_{3} u_{*}^{h}\left(\hat{\mathrm{v}}_{3}\right)=0$. Recalling that $\hat{\mathrm{v}}_{l}=-J_{l} \hat{\mathrm{v}}_{0}, l=1,2,3$ we get

$$
\tilde{C}\left(u_{*}^{h}\right) \hat{\mathrm{v}}_{0}=u_{*}^{h} \hat{\mathrm{v}}_{0}-I_{1} u_{*}^{h} J_{1} \hat{\mathrm{v}}_{1}-I_{2} u_{*}^{h} J_{2} \hat{\mathrm{v}}_{2}-I_{3} u_{*}^{h} J_{3} \hat{\mathrm{v}}_{3}=0 .
$$

Observe that for any fixed $l=1,2,3$ the equations $\tilde{C}\left(u_{*}^{h}\right) \hat{\mathrm{V}}_{0}=0$ and $\tilde{C}\left(u_{*}^{h}\right) J_{l} \hat{\mathrm{v}}_{0}=0$ are equivalent. It remains to note that $\hat{\mathrm{v}}_{0}, J_{1} \hat{\mathrm{v}}_{0}, J_{2} \hat{\mathrm{v}}_{0}$ and $J_{3} \hat{\mathrm{v}}_{0}$ span the horizontal subspace at the point $p \in P$ and therefore $\tilde{C}\left(u_{*}^{h}\right)=0$.

Corollary 3.8. A spinor $u$ is harmonic if and only if the horizontal part $u_{*}^{h}$ of its differential has no quaternion-linear component.

The above Corollary reveals a deep analogy between the Dirac operator and the $\partial$-operator of complex geometry. Indeed, let $\left(Y, I_{Y}\right)$ and $\left(Z, I_{Z}\right)$ be complex manifolds. Assume that a Lie group $G$ acts holomorphically on $Z$ and pick a $G$-principal bundle $\pi^{G}: P_{G} \rightarrow Y$ with a connection. Then the operator $\partial$ can be defined in the usual way, namely

$$
\partial: \Gamma\left(P_{G} \times_{G} Z\right) \stackrel{\nabla}{\longrightarrow} \Omega^{1}(Y) \otimes \Gamma\left(\pi_{!}^{G}\left(u^{*} T Z\right)\right) \rightarrow \Omega^{1,0}(Y) \otimes \Gamma\left(\pi_{!}^{G}\left(u^{*} T Z\right)\right) .
$$

Further, we also have a splitting of the tangent bundle of $P_{G}$ into vertical and horizontal parts: $T P_{G}=\mathcal{V} \oplus \mathcal{H}$. Observe that the bundle $\mathcal{H}$ inherits a complex structure from $T Y$. Then an equivariant map $u$ satisfies the equation $\partial u=0$ if and only if $I_{Z} u_{*}^{h} I_{Y}=u_{*}^{h}$ or, in other words, if and only if the horizontal component has no complex-linear component.

\section{References}

[1] Anselmi, D., And Fré, P. Topological $\sigma$-models in four dimensions and triholomorphic maps. Nuclear Phys. B 416, 1 (1994), 255-300.

[2] Aтiyah, M., And Hitchin, N. The geometry and dynamics of magnetic monopoles. M. B. Porter Lectures. Princeton University Press, Princeton, NJ, 1988.

[3] Bagger, J., And Witten, E. Matter couplings in $N=2$ supergravity. Nuclear Phys. B 222, 1 (1983), 1-10. 
[4] Barth, W. P., Hulek, K., Peters, C. A. M., And Van de Ven, A. Compact complex surfaces, second ed., vol. 4 of Ergebnisse der Mathematik und ihrer Grenzgebiete. 3. Folge. A Series of Modern Surveys in Mathematics [Results in Mathematics and Related Areas. 3rd Series. A Series of Modern Surveys in Mathematics]. Springer-Verlag, Berlin, 2004.

[5] Belcastro, S.-M. Picard lattices of families of $K 3$ surfaces. Comm. Algebra 30, 1 (2002), 61-82.

[6] Boyer, C. P., Galicki, K., And Mann, B. M. Quaternionic reduction and Einstein manifolds. Comm. Anal. Geom. 1, 2 (1993), 229-279.

[7] Chen, J. Complex anti-self-dual connections on a product of CalabiYau surfaces and triholomorphic curves. Comm. Math. Phys. 201, 1 (1999), 217-247.

[8] Chen, J., And Li, J. Quaternionic maps between hyperkähler manifolds. J. Differential Geom. 55, 2 (2000), 355-384.

[9] Donaldson, S. K., And Thomas, R. P. Gauge theory in higher dimensions. In The geometric universe (Oxford, 1996). Oxford Univ. Press, Oxford, 1998, pp. 31-47.

[10] FeIx, B. Hyperkahler metrics on cotangent bundles. J. Reine Angew. Math. 532 (2001), 33-46.

[11] Figueroa-O’Farrill, J. M., Köhl, C., and Spence, B. Supersymmetric Yang-Mills, octonionic instantons and triholomorphic curves. Nuclear Phys. B 521, 3 (1998), 419-443.

[12] Friedman, R. Algebraic surfaces and holomorphic vector bundles. Universitext. Springer-Verlag, New York, 1998.

[13] Fueter, R. Die Funktionentheorie der Differentialgleichungen $\Delta u=0$ und $\Delta \Delta u=0$ mit vier reellen Variablen. Comment. Math. Helv. 7, 1 (1934), 307-330.

[14] Griffiths, P., AND HARRIS, J. Principles of algebraic geometry. Wiley-Interscience [John Wiley \& Sons], New York, 1978. Pure and Applied Mathematics. 
[15] Hitchin, N. J. The self-duality equations on a Riemann surface. Proc. Lond. Math. Soc., III. Ser. 55 (1987), 59-126.

[16] Joyce, D. Hypercomplex algebraic geometry. Quart. J. Math. Oxford Ser. (2) 49, 194 (1998), 129-162.

[17] Kaledin, D. Hyperkaehler structures on total spaces of holomorphic cotangent bundles. In eprint arXiv:alg-geom/9710026 (Oct. 1997).

[18] Maciocia, A. Metrics on the moduli spaces of instantons over Euclidean 4-space. Comm. Math. Phys. 135, 3 (1991), 467-482.

[19] Pidstrygach, V. Y. Hyper-Kähler manifolds and the Seiberg-Witten equations. Tr. Mat. Inst. Steklova 246, Algebr. Geom. Metody, Svyazi i Prilozh. (2004), 263-276.

[20] Sudbery, A. Quaternionic analysis. Math. Proc. Camb. Philos. Soc. 85 (1979), 199-225.

[21] Swann, A. HyperKahler and quaternionic Kahler geometry. Math. Ann. 289, 3 (1991), 421-450.

[22] Taubes, C. H. Nonlinear generalizations of a 3-manifold's Dirac operator. In Trends in mathematical physics (Knoxville, TN, 1998), vol. 13 of AMS/IP Stud. Adv. Math. Amer. Math. Soc., Providence, RI, 1999, pp. $475-486$.

[23] Wang, C. Energy quantization for triholomorphic maps. Calc. Var. Partial Differential Equations 18, 2 (2003), 145-158. 